\title{
Metal-free catalytic asymmetric fluorination of keto esters using a combination of hydrogen fluoride (HF) and oxidant: experiment and computation
}

\author{
Roman Pluta, ${ }^{\dagger}$ Patricia E. Krach, ${ }^{\dagger}$ Luigi Cavallo, ${ }^{\dagger}$ Laura Falivene** and Magnus Rueping* $*^{\dagger+}$ \\ †Institute of Organic Chemistry, RWTH Aachen University, Landoltweg 1, 52074 Aachen, Germany \\ ${ }^{*}$ KAUST Catalysis Center (KCC), King Abdullah University of Science and Technology (KAUST), Thuwal 23955-6900, Saudi Arabia
}

\begin{abstract}
A chiral iodoarene organocatalyst for the catalytic asymmetric fluorination has been developed. The catalyst was used in the asymmetric fluorination of carbonyl compounds providing the products with a quaternary stereocenter with high enantioselectivities. Chiral hypervalent iodine difluoride intermediates were generated in situ by treatment of the catalyst with an oxidant and hydrogen fluoride as fluoride source. As such the $\alpha$-fluorination of a carbonyl compound was achieved with a nucleophilic fluorine source. A combined computational and experimental approach provided insight into the reaction mechanism and the origin of enantioselectivity.
\end{abstract}

KEYWORDS: reaction mechanism, transition state, DFT study, fluorine, hypervalent compounds

\section{INTRODUCTION}

Compounds containing fluorine and fluorinated groups have received increased attention due to their widespread biological and therapeutic properties. ${ }^{1}$ Fluorinated substituents affect the physical, chemical and biological properties of the parent molecules, such as lipophilicity, membrane permeability, biokinetics and biodynamics. Therefore, numerous blockbuster drugs and best-selling agrochemicals as well as many functional materials contain fluorinated moieties. ${ }^{2}$ For these reasons introduction of fluorine and fluorinated substituents have been investigated over decades. Numerous fluorinating reagents have been developed; however, some of them possess certain disadvantages, such as chemical or thermal instability, difficulty in handling, as well as high prices.

Basic strategies for introducing fluorine atoms into nucleophilic positions involve the use of formal-electrophilic fluorine transfer reagents such as NFSI or Selectfluor ${ }^{\circledR}$ (Scheme 1a). ${ }^{3}$ Both their price and bad atom economy limit their potential industrial applications. Elemental fluorine on the other hand is too hazardous to be applied in common laboratory practice and requires moreover specialized equipment.

Recently, oxidative processes have been of particular research interest and nucleophilic fluoride sources such as inexpensive inorganic fluorides or hydrogen fluoride could be applied in combination with an oxidant and an appropriate catalyst. Regarding the development of transition metal-free processes, elegant fluorination protocols were designed using hypervalent iodine species. ${ }^{4}$ In the initial fluorination studies, stoichiometric loadings of hypervalent iodine species were applied in versatile reactions with a wide range of nucleophiles. ${ }^{5,6}$ Catalytic protocols using an aryl iodide, a fluoride and various oxidants were also published, using meta-chloroperbenzoic acid ( $m$ CPBA) or an electric current. ${ }^{7-9}$

However, examples of asymmetric fluorination using hypervalent iodine species are rare. Nevado reported recently the asymmetric aminofluorination of double bonds. ${ }^{10}$ The products were obtained with good selectivities; however, the reaction required stoichiometric amounts of instable hypervalent iodine difluorides. Shibata reported the aminofluorination of double bonds as well as the fluorination of carbonyl compounds applying catalytic amounts of iodoarenes, hydrogen fluoride and an oxidant, however the asymmetric version of the reaction showed only moderate enantioselectivities. ${ }^{8}$ Recently, Jacobsen and co-workers described the enantioselective difluorination of olefins and fluorolactonization reaction using chiral iodoarene catalysts. ${ }^{9}$ At the same time, Gilmour described the catalytic difluorination of terminal olefins. ${ }^{11}$ However, in this case, the development of an asymmetric version was met with moderate success. ${ }^{11}$ Herein we describe the development of superior chiral iodoarene catalysts for the highly selective asymmetric fluorination of carbonyl compounds (Scheme 1b).

a) electrophilic N-F fluorine reagent - previous work

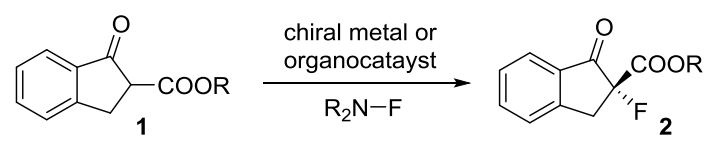

b) nucleophilic fluorine reagent - this work

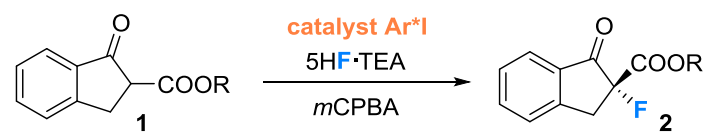

Scheme 1. Catalytic asymmetric fluorinations with a) an electrophilic fluorine reagent; b) nucleophilic fluorine reagent.

\section{RESULTS AND DISCUSSION}

For our initial experiments the indanone based $\beta$-keto ester $\mathbf{1 \mathbf { a } ^ { 3 }}$ was chosen as model substrate together with various catalysts derived from 2-iodoresorcinol and lactate scaffolds (Scheme 2). ${ }^{12}$ Among the different oxidants tested, product formation was observed using $m$ CPBA (see Supporting Information for details), while other oxidants showed no product formation or 
hydroxylation of the substrate only. Hydroxylation of substrate 1a, which originates from unavoidable background reaction of 1a with $m$ CPBA, ${ }^{13}$ was always observed. Additionally, competition between $\mathrm{F}^{-}$and $\mathrm{OH}^{-}$(from residual water) nucleophiles exist in iodoarene catalyzed processes; however, the latter occurs only to a small extent as the observed selectivity of hydroxylated compound was always low (from 50:50 to 55:45 er).

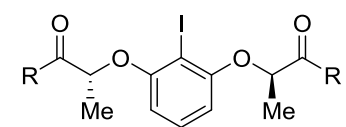

3a: $R=$ OEt $\quad$ 3f: $R=$ NHMes

3b: $R=O^{t} \mathrm{Bu}$

3c: $\mathrm{R}=\mathrm{O}^{\prime} \mathrm{Bu}$

3d: $R=O A d$

3e: $\mathrm{R}=$ OMes

3g: $\mathrm{R}=\mathrm{O}-(R)-1-\mathrm{PhEt}$

3h: $\mathrm{R}=\mathrm{O}-(\mathrm{S})-1-\mathrm{PhEt}$

3i: $\quad R=O-L-m e n t h y l$

3j: $\quad R=O-D-m e n t h y l$

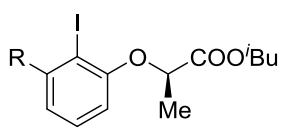

4a: $\mathrm{R}=\mathrm{H}$

4b: $\mathrm{R}=\mathrm{O}^{\prime} \mathrm{Pr}$
${ }^{i B u O O C} \underbrace{\mathrm{B}_{\mathrm{Me}}}_{\overline{\mathrm{Me}}} \mathrm{COO}^{i \mathrm{Bu}}$

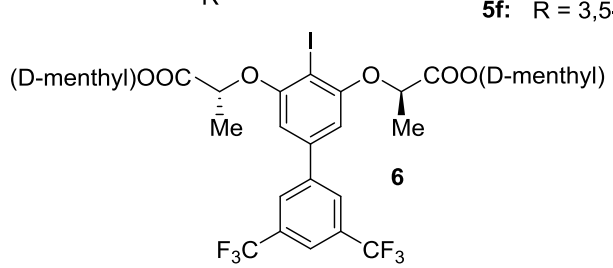

Scheme 2. Catalysts used in this study.

Next, we turned our attention to hydrogen fluoride sources. Experiments using catalyst 3a showed that the choice of hydrogen fluoride source is essential for both good yields and selectivities. The most commonly employed hydrogen fluoride solutions, e.g. aqueous HF, HF in pyridine and triethylamine trihydrofluoride (TEA.3HF) (Table 1, Entries 1-3) provided the desired product in moderate yields. Notably, the TEA.3HF complex produced the product with good selectivity.
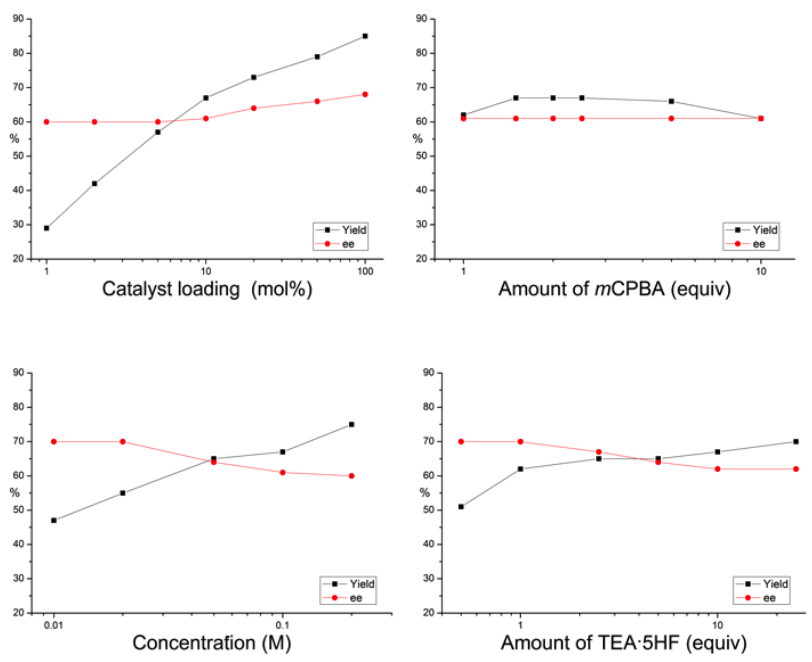

Scheme 3. Examination of reaction parameters using catalyst 3a. (for details see Supporting Information).

Further experiments with other commercial HF sources revealed triethylamine pentahydrofluoride (TEA.5HF) as the best one, offering a compromise between good chemical yield and selectivity of the process. Control experiments showed that in the absence of iodoarene catalyst no fluorinated product formation was observed and only hydroxylation took place. These results clearly demonstrate that the iodoarene catalyst is crucial for the reaction to occur. Further examination of the key reaction parameters such as catalyst loading, amount of $\mathrm{HF}$, concentration and amount of $m \mathrm{CPBA}$ were conducted using catalyst 3a (Scheme 3). A strong impact of the catalyst loading on the yield was observed. This can be explained by the competition with the background process. The selectivity increased only to a small extent when a higher amount of catalyst was used. On the other hand, the amount of $m \mathrm{CPBA}$ did not affect the reaction outcome considerably and both yield and selectivity were comparable in all cases.

Table 1. Optimization of the reaction conditions. ${ }^{a}$

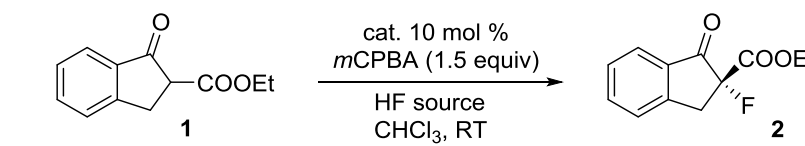

\begin{tabular}{|c|c|c|c|c|c|}
\hline Entry & Catalyst & HF source & Yield $(\%)^{b}$ & $e r^{c}$ & \\
\hline 1 & $3 \mathbf{a}$ & $48 \% \mathrm{HF}_{\mathrm{aq}}$ & 38 & 60: & 40 \\
\hline 2 & $3 \mathbf{a}$ & $70 \% \mathrm{HF}$ in py & 38 & 56: & 44 \\
\hline 3 & $\mathbf{3 a}$ & TEA.3HF & 25 & 85: & 15 \\
\hline 4 & $3 \mathbf{a}$ & TEA $\cdot 5 \mathrm{HF}$ & 65 & 80: & 20 \\
\hline 5 & $3 \mathbf{a}$ & TEAF.4HF & 56 & 79.5: & 20.5 \\
\hline 6 & $3 \mathbf{a}$ & $\mathrm{Me}_{2} \mathrm{O} \cdot 5 \mathrm{HF}$ & 25 & 70: & 30 \\
\hline 7 & 7 & TEA $5 \mathrm{HF}$ & 70 & 63.5: & 36.5 \\
\hline 8 & $3 b$ & TEA $\cdot 5 \mathrm{HF}$ & 59 & 83: & 17 \\
\hline 9 & $3 c$ & TEA $\cdot 5 \mathrm{HF}$ & 68 & 83.5: & 16.5 \\
\hline 10 & 3d & TEA $\cdot 5 \mathrm{HF}$ & 61 & 70: & 30 \\
\hline 11 & $3 \mathbf{e}$ & TEA $\cdot 5 \mathrm{HF}$ & 51 & 87.5: & 12.5 \\
\hline 12 & 3f & TEA.5HF & 39 & 70.5: & 29.5 \\
\hline 13 & $3 g$ & TEA $\cdot 5 \mathrm{HF}$ & 55 & 81: & 19 \\
\hline 14 & $3 h$ & TEA.5HF & 68 & 89: & 11 \\
\hline 15 & $3 \mathbf{i}$ & TEA $\cdot 5 \mathrm{HF}$ & 61 & 83: & 17 \\
\hline 16 & $3 \mathbf{j}$ & TEA $\cdot 5 \mathrm{HF}$ & 65 & 89.5: & 10.5 \\
\hline 17 & $4 \mathbf{a}$ & TEA.5HF & 52 & 60: & 40 \\
\hline 18 & $4 b$ & TEA $\cdot 5 \mathrm{HF}$ & 55 & 65.5: & 34.5 \\
\hline 19 & $5 \mathbf{a}$ & TEA.5HF & $<10$ & 71.5: & 28.5 \\
\hline 20 & $5 b$ & TEA $\cdot 5 \mathrm{HF}$ & 56 & 79: & 21 \\
\hline 21 & $5 c$ & TEA.5HF & 66 & 85.5: & 14.5 \\
\hline 22 & $5 d$ & TEA. $5 \mathrm{HF}$ & 62 & 87: & 13 \\
\hline 23 & $5 e$ & TEA $\cdot 5 \mathrm{HF}$ & 64 & 86: & 14 \\
\hline 24 & $5 f$ & TEA.5HF & 61 & 89.5: & 10.5 \\
\hline 25 & 6 & TEA.5HF & 67 & 95: & 5 \\
\hline $26^{d}$ & 6 & TEA $\cdot 5 \mathrm{HF}$ & 77 & 92: & 8 \\
\hline $27^{e}$ & 6 & TEA $\cdot 5 \mathrm{HF}$ & 39 & 91.5: & 7.5 \\
\hline $28^{f}$ & 6 & TEA.5HF & 61 & 89: & 11 \\
\hline $29^{g}$ & 6 & TEA.5HF & 31 & 95: & 5 \\
\hline $30^{h}$ & 6 & TEA.5HF & $<10$ & 95.5: & 4.5 \\
\hline
\end{tabular}

${ }^{a}$ Reaction conditions: 3a $(0.1 \mathrm{mmol})$, catalyst $(10 \mathrm{~mol} \%)$, HF source (20 equiv of free $\mathrm{HF}$ ) and $m \mathrm{CPBA}(0.15 \mathrm{mmol})$ in chloroform $(1 \mathrm{~mL})$ were stirred in a FEP tube at RT for $18 \mathrm{~h} ;{ }^{b}$ Yields after purification by column chromatography; ${ }^{c}$ er was determined by GC on chiral stationary phase; ${ }^{d} \mathrm{DCM}$ as solvent; ${ }^{e}$ Toluene as solvent; ${ }^{f}$ Acetonitrile as solvent; ${ }^{g}$ Reaction at $0{ }^{\circ} \mathrm{C}$ for $36 \mathrm{~h}$; ${ }^{h}$ Reaction at $-20^{\circ} \mathrm{C}$ for 72 h. 7 : R-1,1'-binaphthyl-2,2'-diiodide. 
Higher HF loading and concentration influenced the reaction outcome resulting in higher yields at the cost of selectivity. The optimized conditions (10 mol \% catalyst, 1.5 equiv $m$ CPBA, concentration $0.1 \mathrm{M}, 5$ equiv TEA.5HF), offering a balance between both chemical yield and selectivity, were chosen for further studies. Next, we turned our attention to the catalyst structure. All catalysts derived from L-(-)-lactic acid ( $S$-lactic acid) tested, as well as $R$-1,1'-binaphthyl-2,2'-diiodide, provided the same enantiomer of the product in excess. Catalysts $\mathbf{4 a}$ and $\mathbf{4 b}$ lacking $\mathrm{C}_{2}$-symmetry proved to be inferior (Table 1, entries 17, 18) and hence we focused on $\mathrm{C}_{2^{-}}$ symmetric structures. Catalysts $\mathbf{3 b}-\mathbf{j}$ obtained by transesterification of the ester group revealed the influence of the steric factors on the selectivity (Table 1, entries 8-16) as bulky esters did not offer sufficient improvement. On the other hand, when chiral alcohols were introduced in the catalyst structure, small matched-mismatched effects were observed (Table 1, entries 13-16). For example catalyst $\mathbf{3 h}$ derived from L-lactic acid and $S$-(-)-1-phenylethanol was superior compared to diastereomeric catalyst $\mathbf{3 g}$ derived from L-lactic acid and $R$ (+)-1-phenylethanol. Among diastereomeric catalysts $\mathbf{3 i}$ and $\mathbf{3 j}$, the best selectivity was obtained with the D-menthyl ester derivative 3j. Next, the electronic effects of differently substituted 2-iodoresorcinols were investigated by applying catalysts 5a-f (Table 1, entries 19-24). Interestingly, electron-donating substituents deteriorated both selectivity and yield. Remarkably, with catalyst $\mathbf{5 a}$ the product was obtained in unexpectedly low yield. This result can be explained by catalyst degradation by the oxidant. On the other hand, electron-withdrawing substituents showed beneficial effects. The best result was obtained using catalyst $5 \mathbf{f}$ bearing a 3,5bis(trifluoromethyl)phenyl group para to the iodine. The catalysts can be separated from the reaction mixture (70-90\% recovery) and reused in the next reactions without any loss of activity or selectivity. Next, catalyst $\mathbf{6}$ which accounts for both steric and electronic effects was prepared and provided the product with excellent selectivity (Table 1, entry 25). Further changes of the reaction conditions, as altering the solvent (Table 1, entries 26-28) or lowering the temperature (Table 1, entries 29-30) did not improve the results.

To get a deeper understanding of the reaction's progress, we studied the kinetics of the asymmetric fluorination reaction of indanone based $\beta$-keto ester 1a (Scheme 4). It is noteworthy, that the ee of the fluorinated product is not changing with the reaction progress.
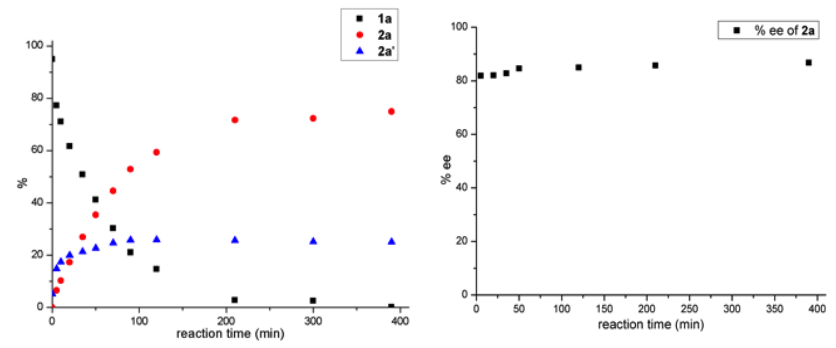

\section{Scheme 4. Kinetics of the asymmetric fluorination.}

With optimized reaction conditions in hand, we decided to explore the utility and applicability of our strategy, applying differently substituted indanone-derived $\beta$-keto esters in reaction with catalysts $\mathbf{3 j}$ and $\mathbf{6}$ (Table 2). A variety of electron- withdrawing and electron-donating substituents were well tolerated, independently of their position in the aromatic ring. Also different ester groups provided the desired products with excellent stereoselectivities. ${ }^{14}$ The absolute configuration of compounds $\mathbf{2 a - d}$ was determined by comparison of the sign of the optical rotation with known samples, suggesting $R$ configuration of the products.

Table 2. Reaction scope of the asymmetric fluorination protocol. $^{a}$

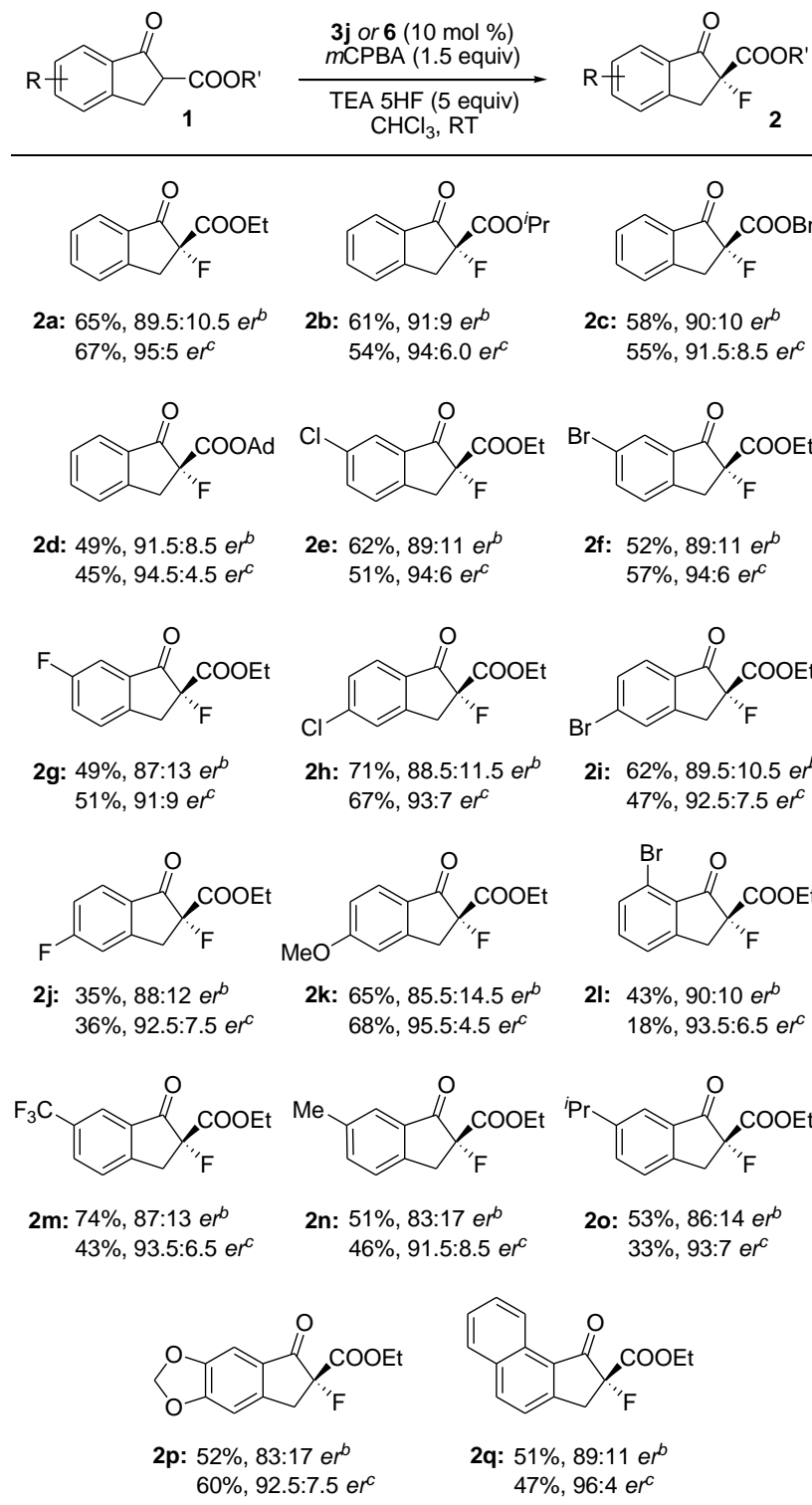

${ }^{a}$ Reaction conditions: 1 (0.1 mmol), catalyst (10 mol \%), TEA.5HF (5 equiv) and $m$ CPBA $(0.15 \mathrm{mmol})$ in chloroform $(1$ $\mathrm{mL}$ ) were stirred in a FEP tube at RT for $4-18 \mathrm{~h}$; isolated yields after column chromatography; er was determined by GC or HPLC on chiral stationary phase. ${ }^{b}$ Reaction with catalyst $\mathbf{3 j}$; ${ }^{c}$ Reaction with catalyst 6 .

The chiral fluorinated products can be functionalized in diverse fashions as demonstrated for compound 2a (Scheme 5). First, modification of the ester group can be performed using amines and alcohols under microwave conditions without loss of enantioselectivity. Reaction with allylamine proceeded 
smoothly and the product 8a was isolated in almost quantitative yield. The Lewis-acid catalyzed reaction with propargylic alcohol provided the product $\mathbf{8 b}$ in good yield. Subsequently, addition of Wittig and Grignard reagents was performed in order to functionalize the ketone moiety. The corresponding products 9 and $\mathbf{1 0}$ were obtained with good yields and the Grignard reaction worked with excellent diastereoselectivity.

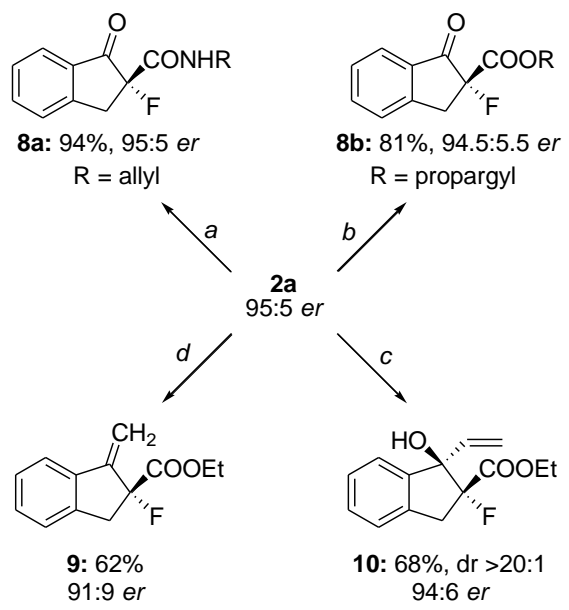

Scheme 5. Functionalization of the product. Reaction conditions: ${ }^{a}$ Allylamine (5 equiv), toluene, 200W MW, $135{ }^{\circ} \mathrm{C}$, $30 \mathrm{~min}$; ${ }^{b}$ Propargyl alcohol (10 equiv), $\mathrm{Bu}_{2} \mathrm{SnO}(5 \mathrm{~mol} \%)$ toluene, 200W MW, $135{ }^{\circ} \mathrm{C}, 45 \mathrm{~min} ;{ }^{c} \mathrm{CH}_{2} \mathrm{CHMgBr}$ (1.1 equiv), THF, $-20{ }^{\circ} \mathrm{C}, 2 \mathrm{~h} ;{ }^{d} \mathrm{Ph}_{3} \mathrm{PCH}_{2}$, toluene, $0{ }^{\circ} \mathrm{C}, 12 \mathrm{~h}$.

Subsequently the scalability of the developed process was investigated with a reduced catalyst loading of $2 \mathrm{~mol} \%$. While the batch reaction proceeded with a lower yield of $43 \%$ (Table 3 , entry 1 ), dropwise addition of the $\beta$-keto ester increased the yield to $67 \%$ (Table 3 , entry 3 ). Slow addition of mCPBA or mCPBA and 1a simultaneously did not further improve the yield (Table 3, entries 2 and 4). Finally, the protocol was scaled up to $1 \mathrm{~g}$ of $\mathbf{1 a}$, providing the product $\mathbf{2 a}$ in good yield and selectivity (Table 3, entry 5).

Table 3. Scaling-up experiments. ${ }^{a}$

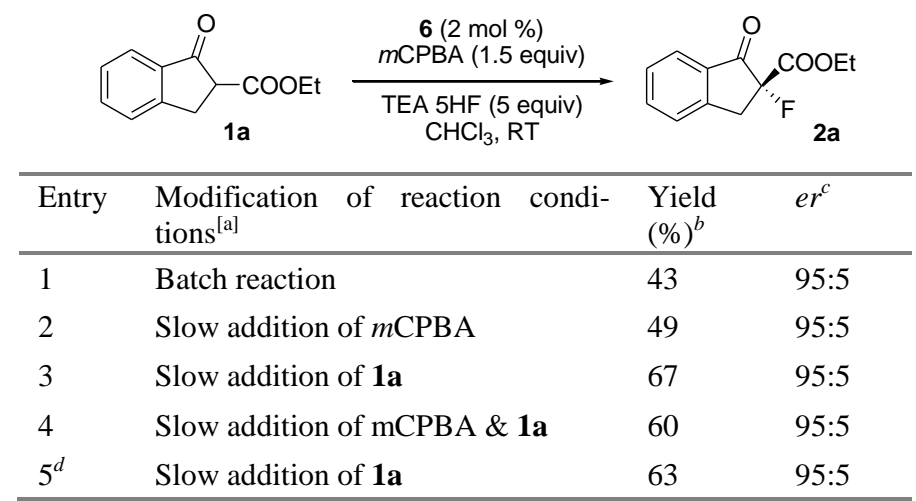

${ }^{a}$ Reaction conditions: 1a $(0.5 \mathrm{mmol}), 6(2 \mathrm{~mol} \%)$, TEA.5HF $(0.5 \mathrm{mmol})$ and $m \mathrm{CPBA}(0.75 \mathrm{mmol})$ in chloroform $(5 \mathrm{~mL})$ were stirred in a FEP tube at RT for $14 \mathrm{~h}$. Indicated reagents were added via syringe pump over $12 \mathrm{~h} .{ }^{b}$ Yields after purification by column chromatography; ${ }^{c}$ er was determined by GC on chiral stationary phase; ${ }^{d}$ Reaction on $5 \mathrm{mmol}$ scale using TEA.5HF (1.5 equiv).
Interestingly, stoichiometric reaction with pre-oxidized hypervalent iodoarene (6-F $\mathbf{F}_{2}$, Scheme 6$)$ showed only traces of the product in the absence of hydrogen fluoride. Upon addition of TEA.5HF, the product was obtained in excellent yield and selectivity. These results are consistent with previous studies and confirm the importance of hydrogen fluoride molecules. ${ }^{5 \mathrm{~b}, \mathrm{e}}$

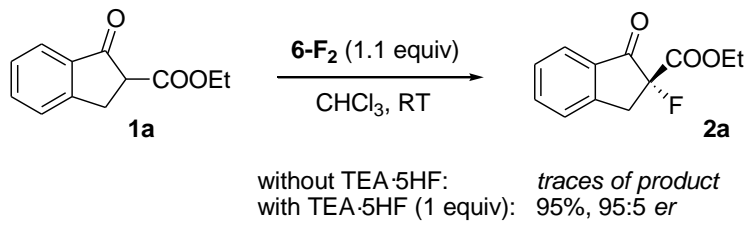

Scheme 6. Stoichiometric studies.

To shed light on the possible reaction mechanism and the origin of enantioselectivity, we performed DFT calculations. ${ }^{15}$ According to the experimental observation we assumed in situ formation and activation of the Ar*-I- $\mathrm{F}_{2}$ by the HF molecule. Ar*-I- $\mathrm{F}_{2}$ can be prepared analyzed (for information SI). The first steps involve the reaction of the enol form of substrate 1a with $\left[\mathrm{Ar}^{*}-\mathrm{I}-\mathrm{F}\right]^{+} /\left[\mathrm{HF}_{2}\right]^{-}$to abstract the $\mathrm{H}$ atom from $\mathbf{1 a}$ via the low energy transition state A-B, with formation of an Obonded hypervalent iodine intermediate $\mathbf{B}$ (Scheme 7). ${ }^{7 \mathrm{a}}$ Intermediate $\mathbf{B}, 27.2 \mathrm{kcal} / \mathrm{mol}$ below the reactant can proceed in a single step to the product, by nucleophilic attack of a fluoride anion to the $\alpha-C$ atom via transition state $\mathbf{B}-\mathbf{2} \mathbf{a}$. Along this path, formation of the $(S)$ enantiomer of the product would be favored, as transition state (pro- $S$ ) B-2a, located at -3.1 $\mathrm{kcal} / \mathrm{mol}$, is favored over transition state (pro- $R$ ) B-2a, at 2.3 $\mathrm{kcal} / \mathrm{mol}$. This conclusion is in disagreement with the experimental selectivity of the observed $(R)$ enantiomer. However, from $\mathbf{B}$ the reaction can more easily proceed via transfer of the $[\mathrm{Ar} *-\mathrm{I}-\mathrm{F}]^{+}$fragment from the carboxylic $\mathrm{O}$ atom to the $\alpha-\mathrm{C}$ atom via transition state $\mathbf{B}-\mathbf{C}$, with formation of an $\alpha-C$ bonded hypervalent iodine intermediate, ${ }^{8,16} \mathbf{C}$ in Scheme 7. Formation of the $(R)$ enantiomer of intermediate $\mathbf{C}$ is favored, as transition state (pro- $R$ ) $\mathbf{B}-\mathbf{C}$, at $-11.1 \mathrm{kcal} / \mathrm{mol}$, is favored over transition state (pro- $S$ ) B-C, at $-9.7 \mathrm{kcal} / \mathrm{mol}$. The energy barrier for this step, calculated as the energy difference between transition state (pro- $R$ ) $\mathbf{B}-\mathbf{C}$ and intermediate $\mathbf{B}$, is 16.1 $\mathrm{kcal} / \mathrm{mol}$. We anticipate that transition state $\mathbf{B}-\mathbf{C}$, which is consistent with previous calculations, ${ }^{17}$ is the enantioselectivity determining transition state, as the resulting intermediate $(R) \mathbf{C}$, located at $-42.3 \mathrm{kcal} / \mathrm{mol}$, is very stable, and the energy barrier for the backward reaction from $\mathbf{C}$ to $\mathbf{B}$, $31.2 \mathrm{kcal} / \mathrm{mol}$, is too high for the working reaction conditions. For this reason, in the following, we only describe the pathway from intermediate $\mathbf{C}$ to the $(R)$ enantiomer of the product.

Again, two pathways are feasible. One pathway corresponds to the reductive elimination of the chiral $\lambda^{3}$ iodane intermediate C via transition state $\mathbf{C - 2 a}$, with a barrier of $21.9 \mathrm{kcal} / \mathrm{mol}$. The other pathway, corresponds to the elimination of $\mathrm{Ar}^{*}-\mathrm{I}$ and formation of an epoxide ring. This occurs via transition state C-D with an energy barrier of $15.3 \mathrm{kcal} / \mathrm{mol}$ only and results in hemiacetale intermediate $\mathbf{D}(-60.3 \mathrm{kcal} / \mathrm{mol})$. Formation of intermediate $\mathbf{D}$ involves inversion of configuration at the $\alpha-C$ atom. Trans opening of the epoxide ring of $\mathbf{D}$ by nucleophilic attack of the fluoride anion, via transition state $\mathbf{D}$ $\mathbf{E}$ and an energy barrier of $13.1 \mathrm{kcal} / \mathrm{mol}$, leads to intermediate $\mathbf{E}$ and restores the $(R)$ configuration at the $\alpha-\mathrm{C}$ atom. Dissociation of the fluoride leads to the experimentally observed $(R)$ enantiomer 2a. We also tried to open the epoxide ring of $\mathbf{D}$ by 
nucleophilic attack of $\mathrm{HF}_{2}^{-}$attack with formation of an $\mathrm{HF}$ molecule, as well as by HF attack with concerted chelation of the fluoride at the carbon ester center. These two pathways proceed via transition states that are 10.0 and $22.9 \mathrm{kcal} / \mathrm{mol}$ higher in energy relative to transition state D-E of Scheme 7 , eliminating these mechanistic alternatives.

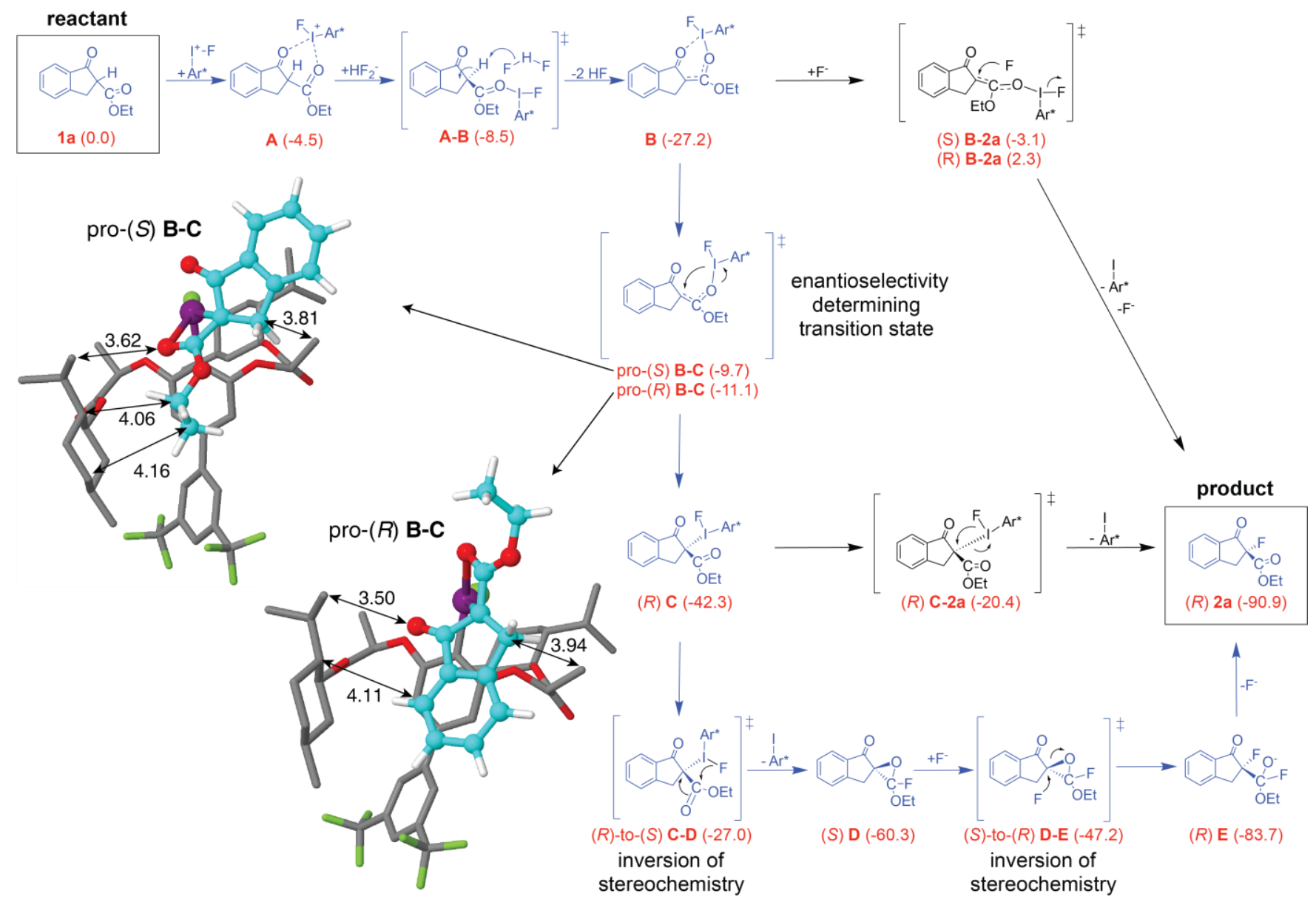

Scheme 7. Proposed reaction mechanism. Free energies in $\mathrm{CH}_{2} \mathrm{Cl}_{2}$ (in kcal/mol). The favored pathway, assembled with a simplified ligand with the isopropyl groups of the D-menthyl moieties replaced by methyl groups, is colored in blue. The structures of the pro$(S)$ and pro- $(R)$ transition states B-C, located using the whole catalyst structure, is reported. C atoms of the substrate are colored in cyan. Selected distances in $\AA$.

Having clarified the whole reaction profile, we focused on the enantioselectivity determining transition state B-C. We first reintroduced the isopropyl groups on the D-menthyl rings, instead of the methyl groups that were used to simplify the catalyst structure while assembling the whole reaction pathway of Scheme 7 . Using the complete catalyst structure we located again the pro- $(R)$ and pro- $(S)$ transition states, and we found again the pro- $(R)$ transition state B-C favored over the pro- $(S)$ transition state. The complete catalyst structure increases the preference for the pro- $(R)$ transition state from 1.4 (see Scheme 7) to $2.3 \mathrm{kcal} / \mathrm{mol}$. The lower energy of the pro(R) transition state can be explained using concepts developed to explain the asymmetric induction of chiral hypercoordinate iodine catalysts for enantioselective transformations. ${ }^{17}$ Consistently with this work, examination of the enantioselectivity determining transition state (Scheme 7) reveals that the unfavored pro- $(S)$ B-C transition state is destabilized by short (and repulsive) distances $(<4.0 \AA$ ) between $\mathrm{C}$ atoms of the substrate and of the catalyst. These distances are systematically larger in the favored pro- $(R)$ transition state. Furthermore, the favored pro- $(R)$ B-C transition state is stabilized by a shorter (and weakly attractive) $\mathrm{C}-\mathrm{H} \cdots \mathrm{O}$ distance between a Oatom of the substrate and $\mathrm{C}-\mathrm{H}$ bonds of the catalyst, compared to the pro- $(S)$ transition state.

\section{CONCLUSIONS}

In summary a new iodoarene catalyst for metal-free asymmetric fluorination of carbonyl compounds was developed. Notably, the presented methodology utilizes inexpensive reagents including hydrogen fluoride as fluorine source to generate the catalytically active iodoarene difluoride species in the presence of $m \mathrm{CPBA}$ as oxidant. The reactions proceed at room temperature under air, tolerate a wide range of functional groups and give the products with excellent enantioselectivities. Mechanistic experiments imply the importance of a hydrogen fluoride containing complex for catalytic activity, which is important for the design of further catalytic asymmetric fluorinations as well as iodoarene catalyzed reactions. DFT calculations provided insight on the whole reaction mechanism and on the origin of enantioselectivity. 


\section{AUTHOR INFORMATION}

\section{Corresponding Author}

*magnus.rueping@rwth-aachen.de; * laura.falivene@gmail.com

\section{Author Contributions}

The manuscript was written through contributions of all authors. / All authors have given approval to the final version of the manuscript.

\section{Notes}

The authors declare no competing financial interests.

\section{ASSOCIATED CONTENT}

Supporting Information. Detailed experimental procedures, spectral data for all compounds, copies of ${ }^{1} \mathrm{H}$ and ${ }^{13} \mathrm{C}$ NMR spectra, as well as cartesian coordinates and energies of all the species are available in the supporting information. This material is available free of charge via the Internet at http://pubs.acs.org.

\section{ACKNOWLEDGMENT}

P.E.K. thanks the Deutsche Bundesstiftung Umwelt for financial support. We also thank Steven Terhorst and Johannes Baues for technical assistance. L.C. thanks the King Abdullah University of Science and Technology (KAUST) for supporting this work. For computer time, this research used the resources of the KAUST Supercomputing Laboratory (KSL) at KAUST.

\section{REFERENCES}

(1) (a) Filler, R.; Kobayashi, Y.; Yugapolskii, L. M. Organofluorine Compounds in Medicinal Chemistry and Biomedical Applications, Elsevier, Amsterdam, 1993. (b) Müller, K.; Faeh, C.; Diederich, F. Science 2007, 317, 1881-1886. (c) Purser, S.; Moore, P. R.; Swallow, S.; Gouverneur, V. Chem. Soc. Rev. 2008, 51, 320-330. (d) Hagmann, W. K. J. Med. Chem. 2008, 51, 4359-4369. (e) Yamazaki, T.; Taguchi, T.; Ojima, I. Fluorine in Medicinal Chemistry and Chemical Biology, Wiley-Blackwell, Chichester, 2009. (f) Manteau, B.; Pazenok, S.; Vors, J.-P.; Leroux, F. R. J. Fluorine Chem. 2010, 131, 140-158. (g) Furuya, T.; Kamlet, A. S.; Ritter, T. Nature 2011, 473, 470-477.

(2) Becker, A. Inventory of industrial fluoro-biochemicals, Eyrolles, Paris, 1996.

(3) For recent reviews, see: (a) Champagne, P. A.; Desroches, J.; Hamel, J.-D.; Vandamme, M.; Paquin, J.-F. Chem. Rev. 2015, 115, 9073-9174. (b) Yang, X.; Wu, T.; Phipps, R. J.; Toste, F. D. Chem. Rev. 2015, 115, 826-870; and references cited therein; early examples on enantioselective fluorinations of $\beta$-keto esters: (c) Shibata, N.; Suzuki, E.; Takeuchi, Y. J. Am. Chem. Soc. 2000, 122, 10728-10729. (d) Cahard, D.; Audouard, C.; Plaquevent, J.-C.; Roques, N. Org. Lett. 2000, 2, 3699-3701. (e) Hamashima, Y.; Yagi, K.; Takano, H.; Tamas, L.; Sodeoka, M. J. Am. Chem. Soc. 2002, 124, 14530-14531. (f) Kim, D. Y.; Park, E. J. Org. Lett. 2002, 4, 545-547. (g) Wang, X.; Lan, Q.; Shirakawa, S.; Maruoka, K. Chem. Commun 2010, 46, 321323. (h) Deng, Q.-H.; Wadepohl, H.; Gade, L. Chem. - Eur. J. 2011, 17, 4922-14928.

(4) (a) Wirth, T. Angew. Chem. Int. Ed. 2005, 44, 3656-3665. (b) Zhdankin, V. V. Arkivoc 2009, 1-62. (c) Parra, A.; Reboredo, S. Chem. Eur. J. 2013, 19, 17244-17260 (d) Singh, F. V.; Wirth, T. Synthesis 2013, 45, 2499-2511. (e) Singh, F. V.; Wirth, T. Chem. Asian J. 2014, 9, 950-971. (f) Dong, D.-Q.; Hao, S.-H.; Wang, Z.-L.; Chen, C. Org. Biomol. Chem. 2014, 12, 4278-4289. (g) Berthiol, F. Synthesis 2015, 47, 587-603. (h) Yoshimura, A.; Zhdankin, V. V. Chem. Rev. 2016, 116, 3328-3345.

(5) Use of iodine difluorides: (a) Zupan, M.; Pollak, A. J. Chem. Soc., Chem. Commun. 1975, 715-716. (b) Hara, S.; Sekiguchi, M.;
Ohmori, A.; Fukuhara, T.; Yoneda, N. Chem. Commun. 1996, 18991900. (c) Hara, S.; Nakahigashi, J.; Ishi-i, K.; Fukuhara, T.; Yoneda, N. Tetrahedron Lett. 1998, 39, 2589-2592. (d) Motherwell, W. B.; Greaney, M. F.; Tocher, D. A. J. Chem. Soc., Perkin Trans. 12002 , 2809-2815. (e) Yoshida, M.; Fujikawa, K.; Sato, S.; Hara, S. Arkivoc 2003, 2003, 36-42. (f) Arrica, M. A.; Wirth, T. Eur. J. Org. Chem. 2005, 395-403. (g) Yu, J.; Tian, J.; Zhang, C. Adv. Synth. Catal. 2010, 352, 531-546. (h) Kitamura, T.; Kuriki, S.; Morshed, M. H.; Hori, Y. Org. Lett. 2011, 13, 2392-2394. (i) Wang, Q.; Zhong, W.; Wei, X.; Ning, M.; Meng, X.; Li, Z. Org. Biomol. Chem. 2012, 10, 8566-8569. (j) Tian, T.; Zhong, W.-H.; Meng, S.; Meng, X.-.B.; Li, Z.-J. J. Org. Chem. 2013, 78, 728-732. (k) Tao, J.; Tran, R.; Murphy, G. K. J. Am. Chem. Soc. 2013, 135, 16312-16315. (1) Kitamura, T.; Muta, K.; Muta, K. J. Org. Chem. 2014, 79, 5842-5846. (m) Liu, G.-Q.; Li, Y.M. J. Org. Chem. 2014, 79, 10094-10109. (n) Nash, T. J.; Pattison, G. Eur. J. Org. Chem. 2015, 3779-3786.

(6) (a) Geary, G. C. ; Hope, E. G.; Singha, K.; Stuart, A. M. Chem. Commun. 2013, 49, 9263-9265. (b) Ilchenko, N. O.; Tasch, B. O. A.; Szabó, K. J. Angew. Chem. Int. Ed. 2014, 53, 12897-12901. (c) Yuan, W.; Szabó, K. J. Angew. Chem. Int. Ed. 2015, 54, 8533-8537. (d) Geary, G. C.; Hope, E. G.; Stuart, A. M. Angew. Chem. Int. Ed. 2015, 54, 14911-14914.

(7) (a) Hara, S.; Hatakeyama, T.; Chen, S.-Q.;. Ishi-i, K; Yoshida, M.; Sawaguchi, M.; Fukuhara, T.; Yoneda, N. J. Fluor. Chem. 1998, 87, 189-192. (b) Kitamura, T.; Muta, K.; Kuriki, S. Tetrahedron Lett. 2013, 54, 6118-6120. (c) Kitamura, T.; Muta, K.; Oyamada, J. J. Org. Chem. 2015, 80, 10431-10436. (d) Banik, S. M.; Mennie, K. M.; Jacobsen, E. N. J. Am. Chem. Soc. 2017, 139, 9152-9155. (e) Kitamura, T.; Miyake, A.; Muta, K.; Oyamada, J. J. Org. Chem. 2017, 82, 11721-11726.

(8) Suzuki, S.; Kamo, T.; Fukushi, K.; Hiramatsu, T.; Tokunaga, E.; Dohi, T.; Kita, Y.; Shibata, N. Chem. Sci. 2014, 5, 2754-2760.

(9) (a) Banik, S. M.; Medley, J. W.; Jacobsen, E. N. J. Am. Chem. Soc. 2016, 138, 5000-5003. (b) Banik, S. M.; Medley, J. W.; Jacobsen, E. N. Science 2016, 353, 51-54. (c) Woerly, E. M.; Banik, S. M.; Jacobsen, E. N. J. Am. Chem. Soc. 2016, 138, 13858-13861. For an example of asymmetric fluorination with fluoride and a chiral organocatalyst, see: (d) Vo, N. T.; Pace, R. D. M.; O'Hara, F.; Gaunt, M. J. J. Am. Chem. Soc. 2008, 130, 404-405.

(10) Kong, W.; Feige, P.; de Haro, T.; Nevado, C. Angew. Chem. Int .Ed. 2013, 52, 2469-2473.

(11) (a) Molnár, I. G.; Gilmour, R. J. Am. Chem. Soc. 2016, 138, 5004-5007. (b) Molnár, I. G.; Thiehoff, C.; Holland, M. C.; Gilmour, R. ACS Catal. 2016, 6, 7167-7173.

(12) This type of catalysts were previously introduced by Ishihara and Fujita; for selected examples, see: (a) Uyanik, M.; Yasui, T.; Ishihara, K. Angew. Chem. Int. Ed. 2010, 49, 2175-2177. (b) Uyanik, M.; Yasui, T.; Ishihara, K. Tetrahedron 2010, 66, 5841-5851. (c) Uyanik, M.; Yasui, T.; Ishihara, K. Angew. Chem. Int. Ed. 2013, 52, 9215-9218. (d) Fujita, M.; Yoshida, Y.; Miyata, K.; Wakisaka, A.; Sugimura, T. Angew. Chem. Int. Ed. 2010, 49, 7068-7071. (e) Fujita, M.; Wakita, M.; Sugimura, R. Chem. Commun. 2011, 47, 3983-3985. (f) Fujita, M.; Mori, K.; Shimogaki, M.; Sugimura, T. Org. Lett. 2012, 14, 1294-1297. (g) Shimogaki, M.; Fujita, M.; Sugimura, T. Eur. J. Org. Chem. 2013, 2013, 7128-7138.

(13) Asahara, H; Nishiwaki, N. J. Org. Chem. 2014, 79, 1173511739.

(14) The reactivity of indanone based $\beta$-diketones is significantly lower under our optimized conditions. The best result obtained was for the reaction of 2-acetyl-2,3-dihydro- $1 \mathrm{H}$-inden-1-one which provided a yield of $27 \%$ with an ee of $29 \%$ for the corresponding product. $\beta$-Keto esters derived from tetralones provided only hydroxylated product under our catalytic conditions and linear substrates were nonreactive.

(15) Geometries were optimized with Gaussian09 package using the PBE0 functional with D3 empirical corrections with BeckeJohnson damping. The electronic configuration of the systems was described with the split-valence SVP basis set for $\mathrm{H}, \mathrm{C}, \mathrm{O}$ and $\mathrm{F}$ while for I we adopted the quasi-relativistic SDD effective core potential with the associated valence basis set. All geometries were characterized as minimum or transition state through frequency calculations. 
The reported free energies were built through single point energy calculations on the PBE/SVP geometries using the M06 functional and the triple- $\zeta$ TZVP basis set for $\mathrm{H}, \mathrm{C}$ and $\mathrm{O}$, augmented with a diffuse basis function for $\mathrm{F}$. Solvent effects were included with the PCM model using $\mathrm{CH}_{2} \mathrm{Cl}_{2}$ as the solvent. To this M06/TZVP electronic energy in solvent, thermal corrections were added from the gasphase frequency calculations at the PBE-D3/SVP level. To reduce the computational cost of the conformational analysis, the isopropyl groups of the D-menthyl moieties were reduced to methyl groups.
(16) Chelli, S.; Troshin, K.; Mayer, P.; Lakhdar, S.; Ofial, A. R.; Mayr, H. J. Am. Chem. Soc. 2016, 138, 10304-10313.

(17) Sreenithya, A.; Patel, C.; Hadad, C. M.; Sunoj, R. B. ACS Catal. 2017, 7, 4189-4196. 
Insert Table of Contents artwork here

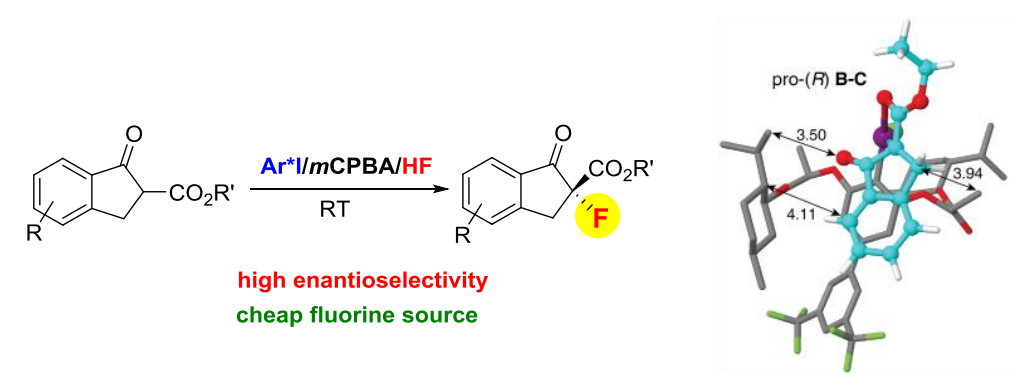

